\title{
Clinical applications of intraoral camera to increase patient compliance - current perspectives
}

This article was published in the following Dove Press journal:

Clinical, Cosmetic and Investigational Dentistry

\section{Kalyana- \\ Chakravarthy Pentapati \\ Hanan Siddiq}

Department of Public Health Dentistry, Manipal College of Dental Sciences, Manipal, Manipal Academy of Higher Education, Manipal, Karnataka, India
Correspondence: Kalyana-Chakravarthy Pentapati

Department of Public Health Dentistry,

Manipal College of Dental Sciences,

Manipal Academy of Higher Education,

Manipal, Karnataka 576I04, India

Tel +91 9916036303

Email drkalyan8I@gmail.com

\begin{abstract}
Technological advancements in the field of dentistry have reformed the concept of photography as a powerful medium of expression and communication. It also offers a spectrum of perception, interpretation, and execution. One of the widespread clinical applications of computerization in dentistry today is the intraoral camera (IOC). It helps in the revelation of the hidden and overlooked defects in teeth and other parts of the cavity. Dental world constitutes of microstructures that have to be recorded in a detailed manner to perform patient education, documentation of records and treatment, illustration of lectures, publication and web connectivity of complicated cases. This review emphasizes the significant applications of IOC in dentistry and its possible impact on patient compliance for dental care.
\end{abstract}

Keywords: applications, camera, compliance, dental, diagnosis, intraoral, teledentistry

\section{Introduction}

Many innovative technologies are being developed that can aid oral health care providers and clinicians to render care effectively. Such novel techniques are generally non-invasive and easy to use with many other benefits for patients and clinicians. One such device that has become an indispensable tool in the provision of oral health care is an intra-oral camera (IOC), which gained its inception in 1987 when the first handpiece was fabricated from the technology adapted from the security industry. ${ }^{1}$

These first dental IOCs were cumbersome and required too much space and at a high cost of about $\$ 40,000$ per unit. Today, IOC systems are about the size of the previous handpiece and cost less than $\$ 5,000$. The cumbersome docking station has been replaced with a USB connectivity and is lightweight and much more comfortable to operate as well as more powerful in taking higher quality images. These major technological upgrades have made the system easy to use, requiring very little training and a standard part of every operatory. ${ }^{2}$

IOC systems consist of video display, processing unit, and intra-oral camera with a light source. Widely ranged features for intra-oral cameras including Macro mode (magnification), curing light for composite, LED lights, picture or video record, fluorescence for detecting various stages of caries, plaque and gingival inflammation are now available. These features in the intra-oral camera can have numerous applications in providing treatment and to communicate with experts regarding diagnosis, treatment decisions, and protocols. Moreover, increasing patient compliance and motivation before, during and after the course of dental 
treatment, patient knowledge and awareness of the various common oral conditions, as well as allowing effective communication between the patient and dentist, remotely of different oral health conditions.

Tremendous advancement in information technology has completely changed the portals of communication between health care personnel and patients. Newer techniques and advancements used to diagnose diseases remotely in real-time and simultaneously seek expert consultations and advice regarding the treatment protocols for various conditions using established telemedicine platforms are now available. In this review, we aim to discuss the various clinical applications of IOC and its possible impact on patient compliance for dental care.

\section{History}

The first real IOC was introduced in the late 80 s. Fuji Optical Systems (Los Gatos, California) received the registered trademark of dental IOC on $7^{\text {th }}$ July 1987 and released DentaCam. Later in 1989, Video Dental Concepts launched a novel IOC device that uses dental endoscopic handpiece. It had dental endoscope, a light source, and a remote head micro camera. Subsequently, many manufacturers modified and enhanced the features that resulted in sophisticated high-end IOC., ${ }^{1,3}$

Initial IOC devices were cumbersome, bulky, expensive, and occupied substantial space in the dental office. These had handpiece, video processor, dedicated computer to process the images and videos that were captured through the devices. Currently, IOC is a small handheld device which is ergonomic, lightweight, comfortable to use, relatively inexpensive and can capture high-quality images and videos that are readily available for the patient and the clinician which can be magnified and viewed. ${ }^{4}$

\section{Working principle of an intra-oral camera} IOC has a sensor located in the handpiece, which can be a charge-coupled device or a complementary metal-oxidesemiconductor sensor. ${ }^{5}$ These sensors receive light which is converted to an electronic signal that is processed by IOC imaging software to produce an image on the computer monitor. Better quality images are obtained when the sensor is placed closer to the lens. The LED lighting provides a continuous source of light to eliminate the need for a flash. ${ }^{6}$ Images captured by most of the IOC are stored in the in-built memory or can be stored in the computer. In-built proprietary software's help in archiving patient photographs and videos. Alternatively, one can use cloud-based storage systems to overcome the limitation of storage space. Development of cordless IOC, which can transmit videos and images in real-time and display in the monitor along with in-built functions like Bluetooth and Wi-Fi connectivity, can increase the portability and performance among the health care professionals. Future IOC software should have in-built teledentistry platforms and should be amenable to communicate through mail, Whatsapp, or any newer methods. Capturing, sharing, transmitting, and archiving of patient images and videos require consent and other relevant procedures. Appropriate care should be taken by the dentist and oral health care professionals to use the information and maintain the confidentiality of the individual.

\section{Literature search}

We have performed a systematic keyword search in two databases (Pubmed and Scopus) up to $1^{\text {st }}$ April 2019. A combination of terms ("intra-oral AND camera" OR "intra AND oral AND camera" OR "intraoral AND camera") was used for both the databases. Articles in English, in-vitro, invivo, clinical studies, trials, and comparative studies were included. Letters, commentaries, short communications, and reviews were excluded. A total of 93 and 178 documents were found in Scopus and Pubmed. All the papers were imported into Rayyan web-based application ${ }^{7}$ and screened for eligibility by two trained examiners. A total of 57 duplicate documents were removed, and 214 records were available for the title and abstract screening. Only records where IOC was used to diagnose, plan the treatment, evaluate the condition, communication, patient education, or any other oral health care applications were included for full-text review.

\section{Applications of IOC}

Applications of IOC can be classified into direct (clinical) and indirect (remote) oral care applications. Clinical or direct applications can be before, during, and after the treatment. Remote applications can be broadly classified into diagnosing, monitoring, and preventive maintenance. Alternatively, they may be classified as those applications used for rendering oral care and those which are useful for increasing patient compliance. The potential clinical applications of IOC have been listed in Figure 1.

\section{Direct or clinical applications of IOC}

It is a common practice for the dentist to examine the oral cavity, perform required investigations, and explain the 


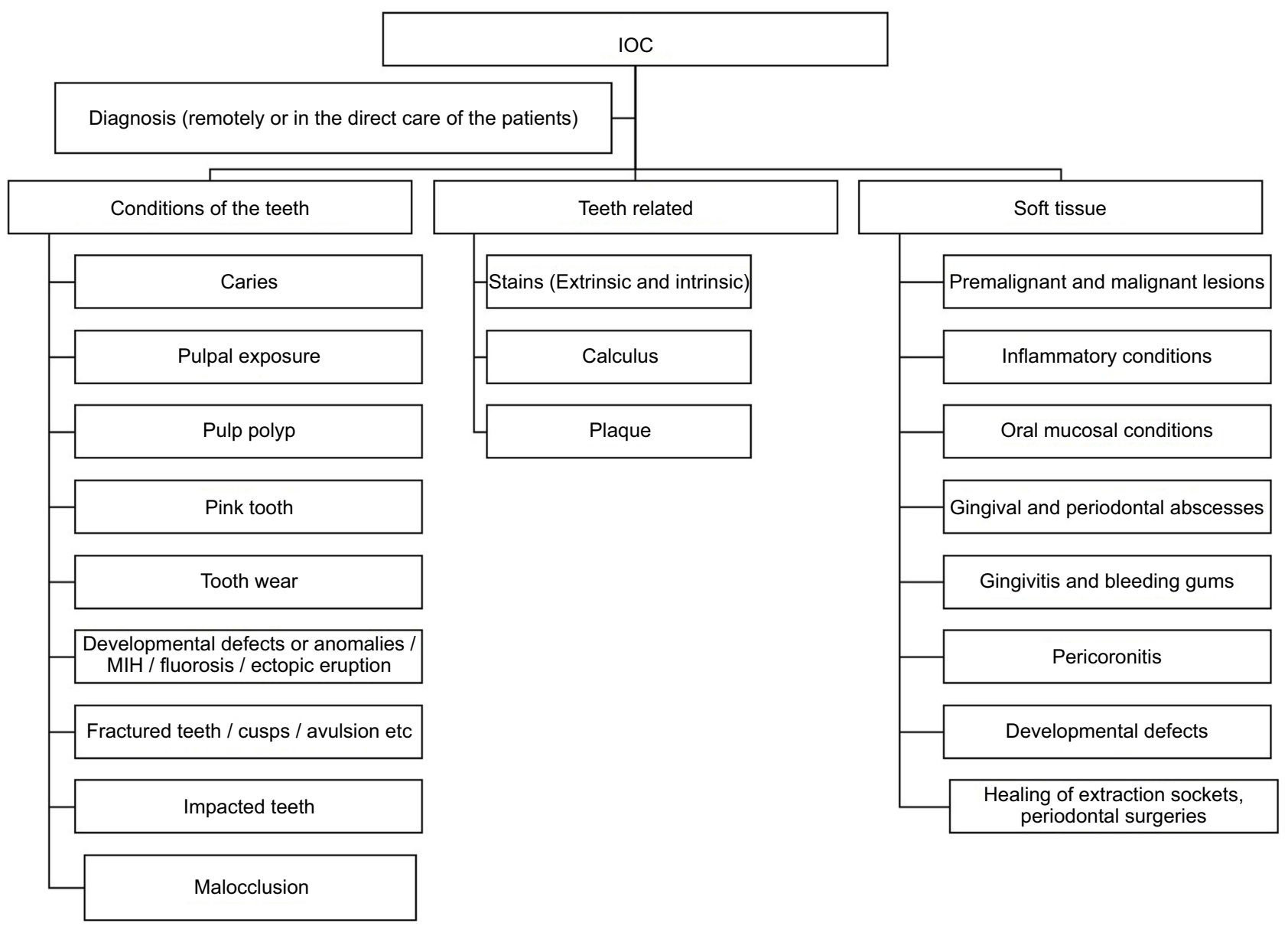

Figure I List of potential clinical applications of Intraoral camera (IOC).

condition of the oral cavity and various treatment modalities to the patient, along with the advantages and disadvantages of the same. In many participants, the scope for understanding the concepts and conditions of the oral cavity and knowledge may not be adequate. This could be challenging for the oral health care provider to convince the patients for available treatment options. Use of IOC can be effective in familiarising the patients with various aspects before, during, and after the treatment.

\section{Diagnosis and treatment of caries and monitoring of restorations}

Studies have shown that caries can be identified reliably with the use of simple IOC. In-vitro studies using videos, recorded by an IOC and simultaneous histological validation showed that IOC examinations significantly increased the detection of occlusal caries. ${ }^{8}$ Another in-vitro study evaluated visual examination, IOC, and operating microscope for detection of occlusal caries, which concluded that IOC and operating microscope improved the detection. ${ }^{9}$ Both of these in-vitro studies reported a rise in false positives.

Similarly, treatment decision making for restorations was compared with a visual examination, IOC, and operating microscope. ${ }^{10}$ It concluded that IOC significantly increased correct treatment decisions compared to unaided visual examination and operating microscope. Boye et al, compared IOC photographs with visual caries assessment and found no significant difference in the caries scores and concluded that photographic assessment could have advantages like reduction in examiner bias, repeatability of assessments, remote screening and can be a permanent record. ${ }^{11}$ However, there can be an increased cost, time taken to capture images, and might need additional software and hardware requirements to digitally archive. A study evaluated children's views on the experience of a visual examination and intra-oral photographs for the diagnosis of dental caries. It concluded that IOC was highly acceptable among children in epidemiological studies. ${ }^{12}$ 
Recently, an LED camera fitted with light-induced fluorescence evaluator (VistaCam, Soprolife, and Soprocare) was developed and clinically validated. The device is not a replacement, but a supplement for the clinician to enhance the diagnostic capabilities. These IOCs have a unique feature called "Cario" mode that provides different colors for healthy, infected, affected active, and arrested dentine. It provides a magnified view of the tooth along with an auto-fluorescent image of the carious enamel and dentin and helps in diagnosis, treatment (excavation), ${ }^{13}$ monitoring of the lesions and restorations (Figure 2). Pits and fissures, which are at risk of developing caries, can also be evaluated at an earlier stage and subsequently sealed. Peycheva and Boteva ${ }^{14}$ in an invitro study reported that Light-Induced Fluorescence (LIF) with SoproLife IOC was more accurate in diagnosing occlusal fissure caries. Soprolife IOC showed to be reliable, and a valid instrument in the diagnosis of caries but may not be superior to the ICDAS. ${ }^{15,16}$ It offers many advantages to the clinicians like a comparison of images before and after the treatments, high-resolution daylight and fluorescent magnified images that can give reliable scores and prevent unnecessary interventions. Shakibaie and Walsh evaluated the effect of contaminations of oral fluids on caries detection by VistaCam IOC. ${ }^{17}$ Presence of blood, and tetracycline staining increased the fluorescent readings of the sound enamel and should be taken into consideration by the clinician. However, moisture or saliva contamination has not affected the scores. JablonskiMomeni et al compared near-infrared imaging system (VistaCam iX Proxi) and conventional digital radiographs for the diagnosis of proximal enamel lesions and found that there was moderate agreement between the methods and found no significant difference. ${ }^{18} \mathrm{~A}$ recent systematic review on LIF with Soprolife IOC has concluded that there was no clear evidence on the detection of occlusal caries lesions and recommended future studies. ${ }^{19}$

Evaluation of dental restorations in terms of marginal defects, fractured restorations, a partial or total loss can also be done using IOC. Signori et al validated the technique of intraoral digital photography captured with IOC against the clinical assessment of the restorations. ${ }^{20}$ There was a fair to moderate agreement for anterior and posterior restorations with higher detection of defects with IOC method. This method has added advantages like permanent archiving, repeatability of the assessments, blinding of the evaluators or outcome assessors, the possibility of the single examiner to evaluate all the restorations even in

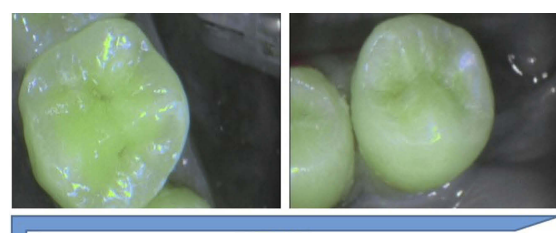

Code 0:

Fissure appears as shiny green; enamel appears sound.

A graphite-pencil-colored thin shine/line-rarely observed.

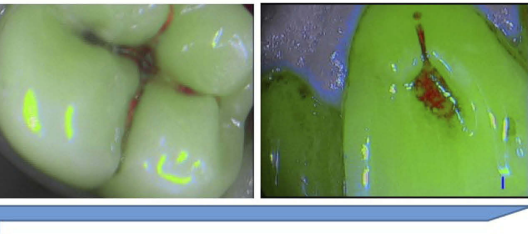

Code 3:

Dark red extended areas are confined to the fissures.

Slight roughness is possible.

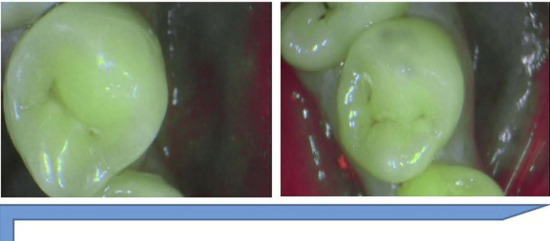

Code 1:

Tiny, thin red shimmer in the pit and fissure system is viewed.

No red dots appeared.

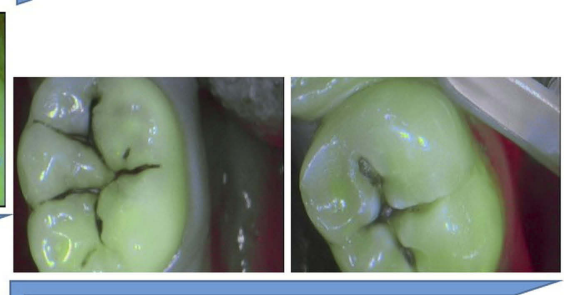

Code 4:

Dark red areas are wider than fissures. Surface roughness occurs. Possibly grey or rough grey zone may be visible.

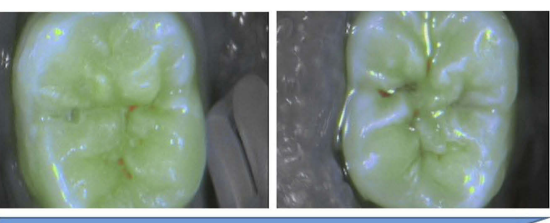

Code 2:

In addition to tiny, thin red shimmer in pits and fissures possibly coming up the slopes darker red spots confined to the fissure are visible.

There was no surface roughness.

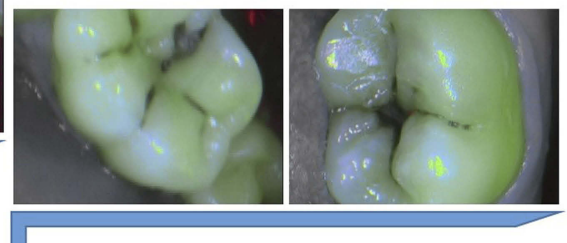

Code 5:

Obvious enamel breakdown with visible dentine was observed.

Reproduced with permission from zeitouny $\mathrm{M}$ et al. ${ }^{9}$

Figure 2 Scores of SOPROLIFE in blue fluorescence mode to detect caries. 
multicentric studies. Monitoring and evaluation of pit and fissure sealants have not been much evaluated. Ferrazzano et al evaluated experimental two-part system (dental arch support and high definition IOC) for capturing standardized images to monitor sealants over a period of time. They concluded that it could be used as standardized and useful methods for sealants retention over time. ${ }^{21}$

Alassaad et al, ${ }^{22}$ used transillumination and IOC for the diagnosis of incomplete cusp fractures, which are mainly diagnosed based on patient symptoms. A technique was proposed to diagnose the incomplete fractures, capture photographs which can be archived and shared with patients. Similarly, they can also be used to diagnose incomplete cuspal fractures and other high-risk areas. Other potential applications of IOC include the ability to diagnose pulpal exposures, pulp polyp, tooth wear for which efficiency has not been evaluated.

\section{Diagnosis of plaque, calculus, and gingival inflammation}

Very few reports have shown the applicability of IOC for the diagnosis of plaque, calculus, and gingival inflammation. These reports have used IOC remotely to diagnose these conditions, along with the many limitations. The use of a plaque disclosing solution is often required as a direct assessment of immature and/or minimal plaque accumulation is difficult to perceive through IOC. Besides, the disclosed immature and minimal plaque may not be seen due to the color clipping effect of the LED lights. Due to the disclosing solution, the early signs of gingivitis can also be masked. A study compared SLR camera and IOC preferences for the assessment of plaque using image analysis. Considering the factors like reliability, simplicity, and flexibility, SLR camera with an image analysis system and the custom frame was more reliable than IOC. $^{23}$ Staudt et al introduced a reproducible, standardized image analysis system for the evaluation of dental plaque using an IOC. It has relatively convenient access to the lingual surfaces, increased objectivity, sensitive to a minor reduction in plaque and can be useful in clinical trials. ${ }^{24}$

Soprocare IOC has a unique feature called "Perio" mode, which helps in overcoming the limitations with conventional IOC. It can immediately highlight the soft and hard deposits around the tooth with different color schemes and magnification levels. It also highlights the marginal inflammation of the gingiva. In a study that evaluated the efficiency "Perio" mode of Soprocare IOC for detecting the plaque and gingival indices, plaque scores using Soprocare were higher and gingival scores were lower than conventional clinical examination. ${ }^{25,26}$ However, these differences were not significant and concluded that "Perio" mode of Soprocare IOC is a reliable tool. Many advantages have been highlighted, such as scoring can be repeated on the pictures, images can be used for patient education, motivation and can be compared with subsequent recall visits to know the effectiveness of treatment and self-efficacy. Shakibaie and Walsh used VistaCam IOC system for comparing calculus and caries fluorescence readings. ${ }^{27}$ Fluorescent readings were significantly higher for calculus than root surface caries and are not affected by saliva and blood, which suggested that VistaCam can be used as an adjunct to clinical examination. The clinician should take into account that there was an overlap in the fluorescent readings for subgingival calculus and root caries.

\section{Endodontics}

The possibility of IOC as a computer-aided diagnostic tool for root canal orifices was evaluated in in-vitro studies. ${ }^{28-31}$ Software programs were developed for standard intraoral or microscopic cameras. These programs were successful in real-time detection of root canal orifices and can be a useful tool for the clinicians and the training of dental students. The application of IOC can guide the clinician with augmented reality and can enhance treatment procedures with high sensitivity. However, an experienced clinician can overcome disadvantages like the higher occurrence of non-existent canal orifices using these software programs and unfavorable dentinal shadows in a few IOC positions.

\section{Orthodontics}

Initial studies were done to evaluate the applications of intra-oral graphic camera called the orthoscan camera. ${ }^{32}$ The Orthoscan camera is an IOC with a mouthpiece and self-illuminated camera which can be placed on the occlusal surfaces. Gholston evaluated the reliability of this device in tooth measurements and found that the tool was highly reliable and gave flat, distortion-free one to one size relationship. ${ }^{33}$

\section{Oral medicine}

Bradley et al, captured high-quality clinical photographs of patients with oral mucosal problems by an IOC to triage the referrals and to evaluate the potential for treatment locally. ${ }^{34}$ Referral patterns were feasible and successful in the management of oral mucosal lesions and particularly useful for the elderly population. However, the study did 
not have a comparison with the clinician's direct provisional diagnosis.

Similarly, a study was done to evaluate the feasibility of a mobile phone camera and WhatsApp application in remote screening oral premalignant lesions. Substantial agreements were seen between actual and remote appbased diagnosis when the lesions were categorized as normal and abnormal, ${ }^{35}$ suggesting a suitable alternative method. IOCs can also be useful in the diagnosis and referral of oral mucosal regions and can be a critical imaging tool in hard to reach areas of the oral cavity. Such potential uses of IOC should be explored in future studies.

\section{Diagnosis of dental hypoplasia, molar hypomineralization, and fluorosis}

Boteva and Peycheva used light-induced fluorescence (LIF) method applied with Soprolife IOC, DIAGNOdent and visual clinical examination to diagnose dental hypoplasia of third molars. ${ }^{36}$ LIF could not differentiate dental hypoplasia and normal structures as both showed green fluorescence. It could only serve as a magnification tool from $30-100 \mathrm{X}$ and can be an aid in clinical diagnosis in hard to reach areas of the oral cavity. Peycheva and Boteva in their studies used light-induced fluorescence (LIF) method applied with Soprolife IOC and DIAGNOdent to diagnose dental fluorosis. LIF method could not differentiate fluorosis and caries lesion. Soprolife IOC "daylight" and "macro mode" can enhance the clinical view with a magnification up to 100 times. $^{37,38}$ Elfrink et al. ${ }^{39}$ validated the use of IOC and photographs to diagnose the molar hypomineralization. It was seen that the photographs captured with IOC had good sensitivity and specificity with a high degree of intra-examiner reliability.

\section{Oral and maxillofacial surgery}

Aziz and Ziccardi discussed the applications of telemedicine using smartphones. Such applications are useful to communicate with surgeons and discuss the treatment plan. ${ }^{40}$ One can readily use smartphones to do a multitude of actions. IOC can be connected and used in place of smartphone cameras and capture images inside the oral cavity with standard illumination. Such a set-up can be used in diagnosis, treatment plan, monitoring, evaluation of healing, dentoalveolar fractures, impactions, pericoronitis, abscess, etc., and the same can be used to communicate with other surgeons. Studies are needed to evaluate the feasibility and reliability of such applications clinically and remotely with teledentistry.

Murphy and Willmot ${ }^{41}$ used an IOC to monitor the size of the fistulas in cleft lip and palate patients. Fistulas were measured using graph paper and caliper technique and images acquired from the IOC. No significant difference was seen between the methods. IOC method is a suitable alternative in monitoring the size of fistulas for clinical and research purposes with multiple advantages like quick, non-invasive, ease of use especially in children, patient education, tracking changes over time, visualization and objective assessment of the fistula, repeatability, and archiving of the data. Ludlow et al used intraoral digital imaging to document the healing of recurrent aphthous lesions. IOC with a modified standardized measurement reference can produce reproducible images of the aphthous lesion and enhances accuracy. ${ }^{42}$

\section{Teledental device for rendering oral care}

Teledentistry can increase access to preventive and diagnostic care for individuals in far-out places by supporting dental hygienists and can allow communication, support, supervision, specialist opinion for oro-facial disorders, and appropriate referral for specialist consultation. ${ }^{43}$ IOC can be used as a teledental device which can transmit the view of the oral cavity using one of the established methods. It was shown to be an effective tool in diagnosis, timely oral health examinations, decreased dental visits, triage, and local treatment of many conditions of the oral cavity.

A field trial on the cost incurred with the use of IOC and teledentistry reported it as a suitable alternative to conventional face-to-face consultations. ${ }^{44}$ An innovative model called "Teledentistry-Assisted, Affiliated Practice for Dental Hygienists" was developed by North Arizona University dental hygiene department. ${ }^{45}$ Individuals can receive preventive services locally, evaluation of oral health by professionals and scheduling necessary treatment, minimal travel, costs, and time off from work or out of school. Alternatively, the participating hygienists are benefitted by delivering preventive services, and the participating dentists are benefitted by appropriate and increased referrals of those patients for whom they can provide the services. Marino et al evaluated the cost analysis of using IOC and teledentistry model to screen residential aged care facility. ${ }^{46}$ It was concluded that asynchronous teledentistry model was a suitable low-cost alternative than face to face or synchronous teledentistry 
examinations. However, the latter might have better outcomes as a two-way communication could be established.

Estai et al have suggested the role of teledentistry in optimizing the referrals to appropriate dental consultants in the field of orthodontics, oral surgery, and medicine. ${ }^{47}$ Such applications can be well executed with appropriate teledentistry platforms and use of IOC. This can reduce inappropriate referrals, validation of referrals, second opinions, support locally-based treatments, and make oral care more accessible to all.

Telehealth assistants have used IOC and teledentistry for screening children for dental caries in the inner-city childcare centers. ${ }^{48}$ Examiners recorded teledentistry examination index for caries similar to the visual examination at baseline and at each teledentistry visits which were used for monitoring prevalence and incidence. Parents were given the printouts of the oral findings of their child as motivation to seek dental care. Regular monitoring was done, and a new referral request was sent to the parent if the treatment was not sought earlier. In a study which evaluated the effectiveness of visual and teledentistry examination using IOC to detect caries among children showed that teledentistry was comparable to visual methods. ${ }^{49}$ The study also concluded that parents who received color printouts of their children's decayed teeth showed more utilization of dental care due to motivation and shared the same with their dentist. Leao and Porter, in their study, used IOC for telediagnosis of oral diseases and concluded that $64 \%$ of the oral health problems were appropriately diagnosed. ${ }^{50}$ IOC was used for screening oral diseases like caries, ${ }^{51,52}$ tooth wear, fluorosis, stains, calculus, plaque through teledentistry with acceptable reliability. ${ }^{52}$

Studies on orthodontic referrals using teledentistry concluded that it could provide better service and optimum use of specialist services. ${ }^{53,54}$ However, this study used video and digital camera to transmit the data. IOC has a potential role in such applications that needs to be assessed by further studies. Berndt et al evaluated the feasibility of teledentistry with IOC for the provision of interceptive orthodontics. ${ }^{55}$ Sufficiently trained dentists can be supervised real-time and can effectively deliver services of interceptive orthodontics. Direct supervision had more improvement than real-time monitoring. However, there was no significant difference. Cook et al conducted an 8 months trial to test a teledentistry prototype software by which general dentists could refer cases of malocclusion with all the relevant clinical and radiographic data. ${ }^{56}$
Six general dentists participated in the study and used different methods like video conferencing, digital camera, flatbed scanners, and IOC to capture the clinical data. All the dentists covered all the aspects of the patients they referred to the specialist.

Rollert et al, ${ }^{57}$ evaluated the IOC and telemedicine for pre-operative assessment for general anesthesia and intubation for oral and maxillofacial surgeries. Most of the patients underwent surgery, and all were identified correctly and concluded that it was a cost-effective alternative in terms of transport. Nickening et al used telemedicine platform via videoconferencing for evaluating preoperative dental implant assessment and found that telemedicine permitted satisfactory evalaution. ${ }^{58}$ The role of IOC needs to be evaluated in such applications.

An in-vitro study evaluated the remote diagnosis of root canal orifices using IOC and teledentistry, concluded that experienced clinicians could guide other clinicians. ${ }^{59}$ The detection rates increased with clinician experience in all situations.

Concepts of telehealth, telemedicine, and teledentistry has been used across the field of oral health for diagnosis, case selection and optimisation of referrals, treatment planning, mentoring, specialist consultation, monitoring of treatment outcomes etc. with diverse gadgets that ranged from digital cameras, video cameras, SLR cameras, mobile phones, intra-oral cameras, web-cameras in conjunction with various software's and adjunct diagnostic modalities in real-time or store and forward. Most of these applications can be successfully implemented with the use of IOCs, but only a few studies in the past used IOCs. Future studies are recommended with the use of IOCs in these areas.

\section{Rendering dental education}

IOC with teledentistry systems can be an effective way to execute clinical training and hands-on continuing dental education for dentists and dental students remotely with low-cost and two-way interaction. ${ }^{43}$ This way cost of the programs can be reduced due to shared resources, regular contact with peers and improved, supervised and quality oral care. Patients with complex oral conditions can be monitored remotely requesting individualized conditionspecific history, examination, investigations, diagnosis, and recommending appropriate management of the condition. Such a system can create and open an endless virtual network of potential oral health service providers. They can network and coordinate the care of one individual 
simultaneously by sharing all the related information to render holistic care.

In dentistry, many procedures require chair-side demonstrations for dental students. It is challenging for students to visualize ongoing intra-oral procedures. To overcome such difficulty, procedures performed by experts can be projected or webcasted or recorded for appropriate training for dental students at all levels. They can be viewed "on the go" or watch at their convenience to get familiarised with all the steps of complex oral treatments. Similarly, training can be facilitated by networking and collaborations with dental institutions, schools, or tertiary health care centers. This way, students would get to see the diverse spectrum of oro-facial disorders and their effective management strategies.

Mustafa et al. ${ }^{60}$ suggested many applications of IOC that can be used in the training of dental students. The routine IOC has options for zoom in, which enhances the view of the operators to see minor details. Such magnification is of a great deal in evaluating cavity preparation, broken teeth and fillings, wear facets on teeth and restorations, evaluation of occlusion, assessment and documentation hard and soft tissue lesions for monitoring, progression and cross-referral with other experts, grading and work assessment of students exercises, training and demonstration of treatment procedures and forensic dentistry.

\section{Forensic dentistry}

IOC can be indispensable in forensic dentistry and can be used to visualize or examine and capture images of the oral cavity. Of the many applications of IOC, dental examination, correct charting, and identifying restorative materials have been studied previously in relation to forensic dentistry. $^{61}$ Shakibaie and Walsh $^{62}$ have evaluated the ability of commercially available intraoral fluorescence camera in identifying dental restorative materials covered with body fluids. There was no significant difference in the scores for any of the restorative material under the influence of moisture, saliva, or blood. On the tooth and root surfaces, there was no significant difference in the presence of moisture while significant difference was seen when it was contaminated with the blood. Similar trend was seen with calculus as well. Also, healthy tooth had significantly different scores than carious teeth and calculus in both moisture and saliva coated samples. Such an IOC can be suitable supplement for mirror, probe and radiographs during forensic dentistry to evaluate teeth and restorations under moisture and saliva. LEDs that can emit fluorescence, white light or infrared illumination can also be of great use in mortuary or field conditions. Blood contamination may nullify the fluorescence, hence during examination such traces can be cleaned and then examined for restorations on to the teeth surfaces. Tsuzuki et al. ${ }^{61}$ also evaluated the usefulness of IOC in forensic inspection in cases of restricted mouth opening due to a multitude of reasons or limited lighting conditions. It was concluded that synthetic photograph generated from multiple photos captured by IOC was effective and in contrast to the conventional photograph where information about the oral conditions is lost due to the inaccessibility.

IOC could capture photos in narrow vestibules and occlusal surfaces of molars in contrast to the conventional photography with intraoral mirrors. IOC used could discriminate the gold and silver fillings, but care has to be exercised when distinguishing caries, discoloration, and tooth-colored restorations. Images thus obtained can be archived, shared to other forensic odontologists, repeat evaluations and for medicolegal purposes. Other potential applications of IOC relevant to forensic dentistry include identification of rugae, fractured teeth or crown, traumatic dental injuries, palate, tongue, missing tooth or teeth, tooth wear, fluorosis, soft tissue lesions or injuries, lip prints, frenum, developmental defects or anomalies, the color of the mucosa, etc. IOC can also be used to evaluate the conditions of the oral cavity, and teeth in archaeological remains non-invasively. Tomczyk et al. ${ }^{63}$ used two fluorescent methods (VistaCam iX proof IOC and DIAGNODent pen) for detection of caries in archaeological remains. Strong correlations were found with VistaCam iX and visual examination with micro-CT. However, the presence of contaminants and calculus can misdiagnose pit and fissure caries with use of VistaCam iX. However, IOC can be used as a screening tool and capture digital images for permanent archiving of the same.

\section{Indirect or remote applications of IOC}

These applications may not be directly linked to the diagnosis or treatment of oral diseases or conditions. Dentists or hygienists can use the IOC to train the patients or individuals to maintain appropriate oral hygiene, proper use of mechanical plaque control aids, demonstration of oral conditions, etc. Alternatively, patients also can use the IOC at home and contact the oral health care providers and 
seek information in real-time or as store and forward method.

It is crucial for the clinician to communicate with the patients about the realistic scenario of the oral cavity and the pros and cons of the treatment proposed. A lot of factors come into play during the process of communication with the patients. Use of pictures and videos captured through IOC of the patient's condition in real-time can reduce the effect of such factors. IOC can help the clinician or oral health provider to communicate with patients and motivate them for treatment, which will increase patient compliance and acceptance that can contribute to the success of the treatment or outcomes.

IOC was shown to be an effective and interactive tool to communicate with patients. ${ }^{64}$ One can use either photograph or live video of the patient's conditions to demonstrate and educate about the oral hygiene and conditions which captures the attention of the patient and understand the pathological processes and boost the correct use of oral hygiene methods. ${ }^{64-66}$ Similarly, an attempt to demonstrate various other conditions like potentially malignant disorders, malignancies, periodontal conditions, pericoronitis, fractured teeth and cusps, incipient caries, deep pits and fissures, pulpal exposure, pulp polyp, and impacted teeth will make the patients understand the characteristics and severity of the disease condition (Figure 3). This way, it is possible to incorporate an approach called "co-diagnosis," which combines the views of patients and oral health care provider in planning the treatment. ${ }^{67}$

Willershausen et al. ${ }^{64}$ evaluated the effectiveness of oral hygiene instructions with and without the use of IOC and concluded that IOC could be used as a tool to reinforce oral hygiene instructions and improve patient compliance. A randomized controlled trial was done to evaluate the effect of IOC on the psychological, behavioral, and clinical parameters among patients with gingivitis. It was seen that IOC significantly improved all the parameters. The use of IOC can reinforce the self-regulation of oral hygiene behaviors like brushing and flossing. The use of photographs and videos captured by the IOC in a dental appointment can contribute to improved gingival health, oral hygiene behaviors, and perceived selfefficacy. ${ }^{66}$ Similarly, an eight-month randomized control trial on the use of IOC and text messages for the control of gingivitis showed that using IOC during consultation and text messages between appointments improves clinical, behavioral, and psychological parameters of periodontal health. $^{68}$

IOC can be used to demonstrate plaque, calculus, and stains on teeth, restorations, prosthesis, and implants to

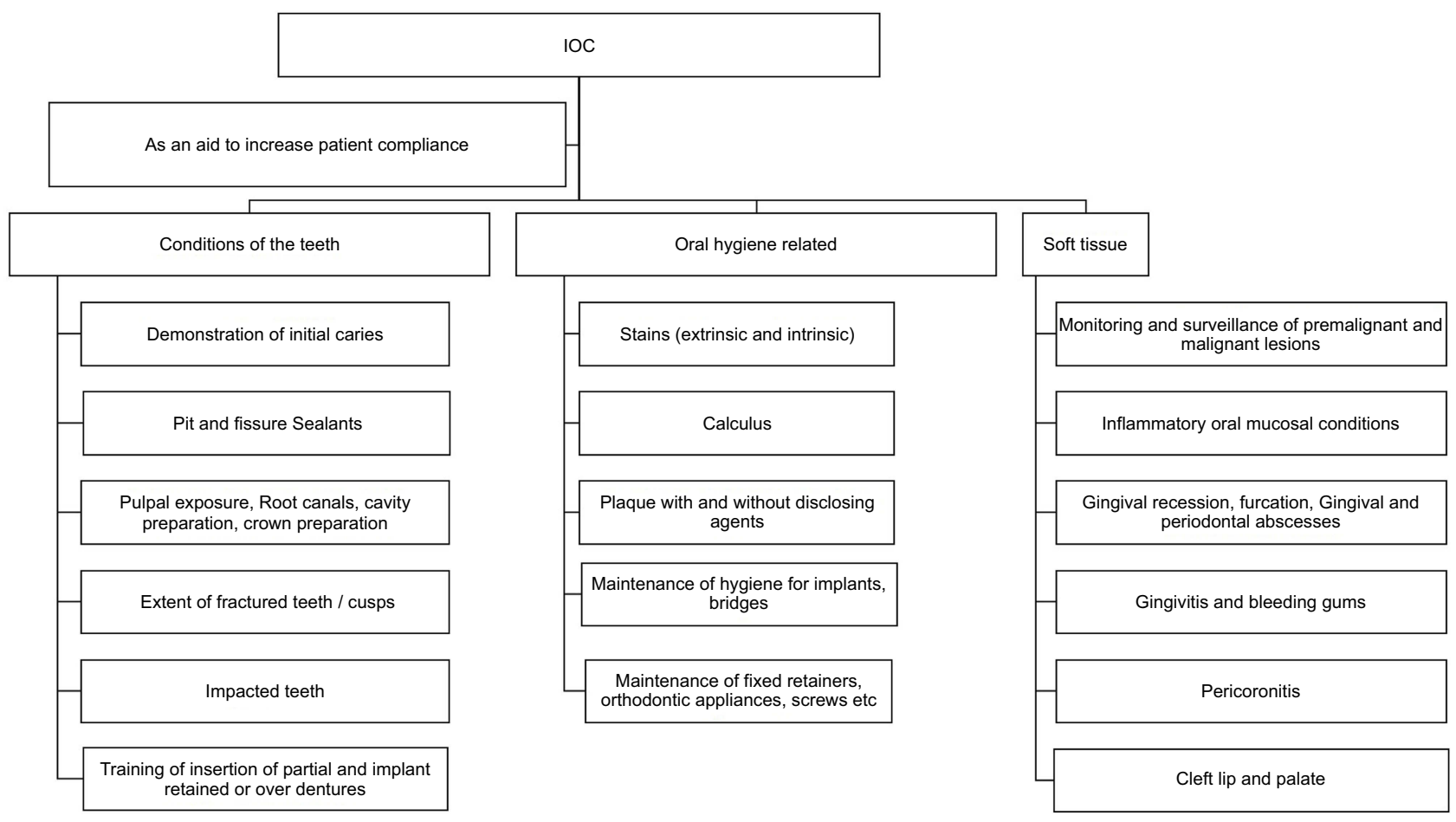

Figure 3 List of applications of Intraoral camera (IOC) as an aid to increase patient compliance. 
patients. It also helps the patients to visualize the areas where biofilm accumulation is more, difficult to remove and inflamed areas. Such a demonstration can optimize the knowledge, attitudes, and practices about oral hygiene and motivate them for the use of appropriate oral hygiene methods and correct interdental aids as demonstrated by the oral health care provider. IOCs like SoproCARE and SoproLIFE have advanced features which can highlight the areas of gingival inflammation, plaque, early caries lesions with the help of their "PERIO mode" and "CARIO mode" respectively. This will help to reinforce the patients about the need for home care and indirectly reduces the burden of in-office periodontal treatment. A randomized trial to evaluate the role of IOC along with supervised brushing among children, showed that IOC could be effective reinforcement tool for reduction of plaque scores. ${ }^{69}$

IOC can be used for periodic self-assessment by the patient regarding the effectiveness of self-care and tissue status around dental implants. Also, images or videos can be transmitted to the healthcare professional. Subjects can communicate and locate the areas of food lodgment, redness, swelling, or any other signs which could be prevented at an early stage. ${ }^{70}$ Peri-implant conditions like plaque, mucosal assessment, width of peri-implant keratinised tissue, suppuration, occlusal evaluation, implant stability or mobility can be evaluated remotely and can be demonstrated to the patient in real-time through the use of IOC. At home implant hygiene requires additional care like use of soft brushes, end-tufted brush, foam tips, floss, plastic-coated interproximal brushes, wooden picks, irrigation systems and special techniques of usage (interproximal/circumferential flossing) which can be demonstrated with the use of IOC.

IOC can also be used to train the individual for placement and removal of cast partial dentures, acrylic dentures, complete dentures, implant retained dentures. Maintenance of hygiene by the use of interdental aids can be demonstrated with the help of IOC in areas below the fixed partial denture and adjacent teeth of the abutment.

Involvement of the patient increases when they see their oral conditions while it is being discussed and communicated by the dental professional. Measures such as the use of floss, floss threader, power floss, use of interdental brushes, implant maintenance, sub-gingival irrigation can be demonstrated in real-time to the patient. Repeated reinforcements can be done at the follow-up visits until the optimum level of oral hygiene is achieved among the individuals. Such a process can be more emphasized in children and adolescents, older individuals, high-risk groups, patients with a fixed prosthesis, pontics, implants, and those undergoing orthodontic treatment.

Similarly, training sessions can be conducted to the patients about the insertion of sophisticated prosthesis or insertion and activation of orthodontic appliances. Patients can be explained their pivotal role in the management of biofilm and can be taught the possible adverse outcomes of non-compliance for the same. Use of IOC can aid the clinician and patients in regular monitoring could be initiated by checking how effective the individual is performing home care maintenance, and the same can be shown, demonstrated in real-time which also can be digitized and archived for permanent record.

IOC can also be used to increase patient compliance by directly watching the treatment in real-time or recorded versions for patient familiarity and understanding the various stages of dental treatment, especially among children and anxious patients. Similarly, IOC can be used by the patients to seek expert advice on different oral conditions, monitoring of the oral conditions, healing directly from home using any of the teledentistry concepts with minimal training.

\section{Conclusion}

It can be concluded that IOC has diverse applications in oral health care and can effectively assist dentist, dental hygienists, and oral health care providers. Also, the compliance of the patient can be increased by the use of IOCs in diagnosis, treatment planning, goal setting, oral hygiene instructions, and maintenance. However, studies are scant in this area, and a precise understanding of the mechanisms of IOC by which patient compliance can be increased is not very clear for many other potential applications.

\section{Disclosure}

The authors report no conflicts of interest in this work.

\section{References}

1. McCann D, Fisch S. Dental technology: knocking at high-tech's door. $J$ Am Dent Assoc. 1989;118(3):285-294. doi:10.14219/JADA. ARCHIVE.1989.0095

2. Snyder TL. The Intraoral Camera: A Popular Computerized Tool. $J$ Am Dent Assoc. 1995;126:177-178.

3. History of the Intraoral Dental Camera | Favoriteplus.com Blog. Available from: https://www.favoriteplus.com/blog/history-intraoraldental-camera/. Accessed July 21, 2019. 
4. The Story behind Intraoral Cameras Lensiora. Available from: https://www.lensiora.com/the-history-of-intraoral-cameras/\#abh about. Accessed July 21, 2019.

5. Desai V, Bumb D. Digital dental photography: a contemporary revolution. Marwah N, Toumba K, eds. Int J Clin Pediatr Dent. 2013;6 (3):193-196. doi:10.5005/jp-journals-10005-1217

6. Panning into the future: a wide angle view of intraoral cameras helps determine your needs | Registered Dental Hygienist. Available from: https://www.rdhmag.com/career-profession/students/article/ 16409320/panning-into-the-future-a-wide-angle-view-of-intraoralcameras-helps-determine-your-needs. Accessed July 21, 2019.

7. Ouzzani M, Hammady H, Fedorowicz Z, Elmagarmid A. Rayyan-a web and mobile app for systematic reviews. Syst Rev. 2016;5(1):210. doi:10.1186/s13643-016-0384-4

8. Forgie AH, Pine CM, Pitts NB. The assessment of an intra-oral video camera as an aid to occlusal caries detection. Int Dent J. 2003;53 (1):3-6.

9. Erten H, Uçtasli MB, Akarslan ZZ, Uzun O, Baspinar E. The assessment of unaided visual examination, intraoral camera and operating microscope for the detection of occlusal caries lesions. Oper Dent. 2005;30(2):190-194.

10. Erten H, Uçtasli MB, Akarslan ZZ, Uzun O, Semiz M. Restorative treatment decision making with unaided visual examination, intraoral camera and operating microscope. Oper Dent. 2006;31(1):55-59. doi:10.2341/04-173

11. Boye U, Willasey A, Walsh T, Tickle M, Pretty IA. Comparison of an intra-oral photographic caries assessment with an established visual caries assessment method for use in dental epidemiological studies of children. Community Dent Oral Epidemiol. 2013;41(6):526-533. doi:10.1111/cdoe.12049

12. Boye U, Foster GRK, Pretty IA, Tickle M. Children's views on the experience of a visual examination and intra-oral photographs to detect dental caries in epidemiological studies. Community Dent Health. 2012;29(4):284-288.

13. Gugnani N, Srivastava N, Gugnani S, Pandit I, Gupta M. Light induced fluorescence evaluation: a novel concept for caries diagnosis and excavation. J Conserv Dent. 2011;14(4):418. doi:10.4103/09720707.87216

14. Peycheva K, Boteva E. A comparison of different methods for fissure caries detection. Acta Medica Bulg. 2016;43(1):30-38. doi:10.1515/ amb-2016-0004

15. Zeitouny M, Feghali M, Nasr A, et al. SOPROLIFE system: an accurate diagnostic enhancer. Sci World J. 2014:924741. doi:10.1155/2014/924741

16. Theocharopoulou A, Lagerweij MD, van Strijp AJ. Use of the ICDAS system and two fluorescence-based intraoral devices for examination of occlusal surfaces. Eur J Paediatr Dent. 2015;16 (1):51-55.

17. Shakibaie F, Walsh LJ. Effect of oral fluids on dental caries detection by the VistaCam. Clin Exp Dent Res. 2015;1(2):74-79. doi:10.1002/ cre 2.13

18. Jablonski-Momeni A, Jablonski B, Lippe N. Clinical performance of the near-infrared imaging system VistaCam iX Proxi for detection of approximal enamel lesions. BDJ Open. 2017;3(1):17012. doi:10.1038/bdjopen.2017.12

19. Doméjean S, Rongier J, Muller-Bolla M. Detection of occlusal carious lesion using the soprolife ${ }^{\circledR}$ camera: a systematic review. $J$ Contemp Dent Pract. 2016;17(9):774-779.

20. Signori C, Collares K, Cumerlato CBF, Correa MB, Opdam NJM, Cenci MS. Validation of assessment of intraoral digital photography for evaluation of dental restorations in clinical research. J Dent. 2018;71:54-60. doi:10.1016/j.jdent.2018.02.001

21. Ferrazzano GF, Orlando S, Cantile T, et al. An experimental in vivo procedure for the standardised assessment of sealants retention over time. Eur J Paediatr Dent. 2016;17(3):176-180.
22. Alassaad SS. Incomplete cusp fractures: early diagnosis and communication with patients using fiber-optic transillumination and intraoral photography. Gen Dent. 2011;59(2):132-135.

23. Smith RN, Rawlinson A, Lath DL, Brook AH. A digital SLR or intraoral camera: preference for acquisition within an image analysis system for measurement of disclosed dental plaque area within clinical trials. J Periodontal Res. 2006;41(1):55-61. doi:10.1111/j.16000765.2005.00841.x

24. Staudt CB, Kinzel S, Hassfeld S, Stein W, Staehle HJ, Dörfer CE. Computer-based intraoral image analysis of the clinical plaque removing capacity of 3 manual toothbrushes. J Clin Periodontol. 2001;28(8):746-752.

25. Rechmann P, Liou SW, Rechmann BM, Featherstone JD. SOPROCARE - $450 \mathrm{~nm}$ wavelength detection tool for microbial plaque and gingival inflammation: a clinical study. Proc. SPIE 8929, Lasers in Dentistry XX, 892906 (27 February 2014). doi:10.1117/12.2047275

26. Rechmann P, Liou SW, Rechmann BMT, Featherstone JDB. Performance of a light fluorescence device for the detection of microbial plaque and gingival inflammation. Clin Oral Investig. 2016;20(1):151-159. doi:10.1007/s00784-015-1481-9

27. Shakibaie F, Walsh LJ. Dental calculus detection using the VistaCam. Clin Exp Dent Res. 2016;2(3):226-229. doi:10.1002/cre2.42

28. Brüllmann DD, Alvarez P, Willershausen B. Recognition of root canal orifices in video sequences as a future support system during endodontic treatment. $J$ Endod. 2009;35(10):1400-1403. doi:10.1016/j.joen.2009.06.013

29. Brüllmann D, Briseño B, Willershausen B, d'Hoedt B. Fast algorithm for real-time detection of root canal orifices in video images. Int $J$ Comput Dent. 2006;9(4):299-306.

30. Brüllmann DD, Weichert CID, Daubländer M. Intraoral cameras as a computer-aided diagnosis tool for root canal orifices. J Dent Educ. 2011;75(11):1452-1457.

31. Bruellmann DD, Tjaden H, Schwanecke U, Barth P. An optimized video system for augmented reality in endodontics: a feasibility study. Clin Oral Investig. 2013;17(2):441-448. doi:10.1007/s00784-012-0718-0

32. Chanda LH. The orthoscan camera. J Clin Orthod. 1973;7(6):363367.

33. Gholston LR. Reliability of an intraoral camera: utility for clinical dentistry and research. Am J Orthod. 1984;85(1):89-93.

34. Bradley M, Black P, Noble S, Thompson R, Lamey PJ. Application of teledentistry in oral medicine in a community dental service, N. Ireland. Br Dent J. 2010;209(8):399-404. doi:10.1038/sj.bdj.2010.928

35. Vinayagamoorthy K, Acharya S, Kumar M, Pentapati KC, Acharya S. Efficacy of a remote screening model for oral potentially malignant disorders using a free messaging application: a diagnostic test for accuracy study. Aust J Rural Health. 2019;ajr.12496. doi:10.1111/ ajr. 12496

36. Boteva E, Peycheva K. Detection of dental hypoplasia - ability of fluorescence methods. Acta Medica Bulg. 2013;40(1):61-64.

37. Peycheva K, Boteva E. Methods for diagnosing dental fluorosis: quantitative laser fluorescence and light-induced fluorescence. Acta Medica Bulg. 2013;40(1):53-60.

38. Peycheva K, Boteva E. A clinical study on dental fluorosis with lightinduced fluorescence. Int J Sci Res. 2015;4(3):1239-1241.

39. Elfrink MEC, Veerkamp JSJ, Aartman IHA, Moll HA, Ten Cate JM. Validity of scoring caries and primary molar hypomineralization (DMH) on intraoral photographs. Eur Arch Paediatr Dent. 2009;10 (Suppl 1):5-10.

40. Aziz SR, Ziccardi VB. Telemedicine using smartphones for oral and maxillofacial surgery consultation, communication, and treatment planning. J Oral Maxillofac Surg. 2009;67(11):2505-2509. doi:10.1016/j.joms.2009.03.015

41. Murphy TC, Willmot DR. Image analysis of oronasal fistulas in cleft palate patients acquired with an intraoral camera. Plast Reconstr Surg. 2005;115(1):31-37. 
42. Ludlow JB, Kutcher MJ, Samuelson A. Intraoral digital imaging documenting recurrent aphthous ulcer healing in 2-octyl cyanoacrylate versus sham-treated lesions. Oral Surg Oral Med Oral Pathol Oral Radiol Endod. 2000;89(4):425-431.

43. Fricton J, Chen H. Using teledentistry to improve access to dental care for the underserved. Dent Clin North Am. 2009;53(3):537-548. doi:10.1016/j.cden.2009.03.005

44. Marino R, Hopcraft M, Tonmukayakul U, et al. Teleconsultation/ telediagnosis using teledentistry technology: a pilot feasibility study. Int J Adv Life Sci. 2014;6:291-299.

45. Summerfelt FF. Teledentistry-assisted, affiliated practice for dental hygienists: an innovative oral health workforce model. J Dent Educ. 2011;75(6):733-742.

46. Mariño R, Tonmukayakul U, Manton D, Stranieri A, Clarke K. Costanalysis of teledentistry in residential aged care facilities. $J$ Telemed Telecare. 2016;22(6):326-332. doi:10.1177/1357633X15608991

47. Estai M, Kruger E, Tennant M. Optimizing patient referrals to dental consultants: implication of teledentistry in rural settings. Australas Med J. 2016;09(07):249-252. doi:10.4066/AMJ.2016.2696

48. Kopycka-Kedzierawski DT, Billings RJ. Teledentistry in inner-city child-care centres. J Telemed Telecare. 2006;12(4):176-181. doi:10.1258/135763306777488744

49. Kopycka-Kedzierawski DT, Billings RJ. Comparative effectiveness study to assess two examination modalities used to detect dental caries in preschool urban children. Telemed $J$ E Health. 2013;19 (11):834-840. doi:10.1089/tmj.2013.0012

50. Leao JC, Porter SR. Telediagnosis of oral disease. Braz Dent J. 1999;10(1):47-53.

51. Patterson S, Botchway C. Dental screenings using telehealth technology: a pilot study. J Can Dent Assoc. 1998;64(11):806-810.

52. Pentapati KC, Mishra P, Damania M, Narayanan S, Sachdeva G, Bhalla G. Reliability of intra-oral camera using teledentistry in screening of oral diseases - pilot study. Saudi Dent J. 2017;29 (2):74-77. doi:10.1016/j.sdentj.2017.03.002

53. Stephens C, Cook J, Mullings C. Orthodontic referrals via TeleDent Southwest. Dent Clin North Am. 2002;46(3):507-520.

54. Mandall NA, O’Brien KD, Brady J, Worthington HV, Harvey L. Teledentistry for screening new patient orthodontic referrals. Part 1: a randomised controlled trial. Br Dent J. 2005;199(10):659-662. doi:10.1038/sj.bdj.4812930

55. Berndt J, Leone P, King G. Using teledentistry to provide interceptive orthodontic services to disadvantaged children. Am J Orthod Dentofac Orthop. 2008;134(5):700-706. doi:10.1016/j.ajodo.2007.12.023

56. Cook J, Mullings C, Vowles R, Ireland R, Stephens C. Online orthodontic advice: a protocol for a pilot teledentistry system. J Telemed Telecare. 2001;7(6):324-333. doi:10.1258/1357633011936958
57. Rollert MK, Strauss RA, Abubaker AO, Hampton C. Telemedicine consultations in oral and maxillofacial surgery. J Oral Maxillofac Surg. 1999;57(2):136-138.

58. Nickenig H-J, Wichmann M, Schlegel A, Eitner S. Use of telemedicine for pre-implant dental assessment - a comparative study. $J$ Telemed Telecare. 2008;14(2):93-97. doi:10.1258/jtt.2007.070806

59. Brüllmann D, Schmidtmann I, Warzecha K, d'Hoedt B. Recognition of root canal orifices at a distance - a preliminary study of teledentistry. $J$ Telemed Telecare. 2011;17(3):154-157. doi:10.1258/jtt.2010. 100507

60. Mustafa NS, Kashmola MAH, Mustafa Al-Ahmad BE. The use of intraoral camera in the assessment of students requirement (work). Eur J Sci Res. 2012;69(4):489-493.

61. Tsuzuki T, Ueno A, Kajiwara M, et al. Evaluation of intraoral CCD camera for dental examination in forensic inspection. Leg Med (Tokyo). 2002;4(1):40-46.

62. Shakibaie F, Walsh LJ. Fluorescence imaging of dental restorations using the VistaCam intra-oral camera. Aust J Forensic Sci. 2017;1-9. doi:10.1080/00450618.2017.1304991

63. Tomczyk J, Komarnitki J, Zalewska M, Lekszycki T, OlczakKowalczyk D. Fluorescence methods (VistaCam iX proof and DIAGNODent pen) for the detection of occlusal carious lesions in teeth recovered from archaeological context. Am J Phys Anthropol. 2014;154(4):525-534. doi:10.1002/ajpa.22542

64. Willershausen B, Schlösser E, Ernst CP. The intra-oral camera, dental health communication and oral hygiene. Int Dent J. 1999;49(2):95-100.

65. Ahmad I. Digital dental photography. Part 2: purposes and uses. $\mathrm{Br}$ Dent J. 2009;206(9):459-464. doi:10.1038/sj.bdj.2009.366

66. Araújo M-R, Alvarez M-J, Godinho CA, Pereira C. Psychological, behavioral, and clinical effects of intra-oral camera: a randomized control trial on adults with gingivitis. Community Dent Oral Epidemiol. 2016;44(6):523-530. doi:10.1111/cdoe.12245

67. Blique M, Grosse S. Contribution of fluorescence and selective chromatic amplification in daily preventive dentistry practise. Dental Asia. 2013;52-55.

68. Araújo M, Alvarez M, Godinho CA, Roberto MS. An eight-month randomized controlled trial on the use of intra-oral cameras and text messages for gingivitis control among adults. Int J Dent Hyg. 2019;17(3):idh.12391. doi:10.1111/idh.12391

69. Machale P, Hegde-Shetiya S, Kakodkar P, Shirahatti R, Agarwal D, Kakade S. Effect of using an intra-oral camera as a reinforcement tool for plaque control in a supervised toothbrushing program: an interventional study. J Indian Assoc Public Heal Dent. 2016;14 (2):110. doi:10.4103/2319-5932.183807

70. Gulati M, Govila V, Anand V, Anand B. Implant maintenance: a clinical update. Int Sch Res Not. 2014;2014:908534. doi:10.1155/ $2014 / 908534$
Clinical, Cosmetic and Investigational Dentistry

\section{Publish your work in this journal}

Clinical, Cosmetic and Investigational Dentistry is an international, peer-reviewed, open access, online journal focusing on the latest clinical and experimental research in dentistry with specific emphasis on cosmetic interventions. Innovative developments in dental materials, techniques and devices that improve outcomes and patient satisfaction and preference will be highlighted. The manuscript management system is completely online and includes a very quick and fair peer-review system, which is all easy to use. Visit http://www.dovepress.com/testimonials.php to read real quotes from published authors. 